misuse of antimicrobial drugs and to improve antibiotic use in healthcare systems. Along with professional societies and other stakeholders, the CDC already has started to prepare clinical guidelines for health professionals on how best to use antimicrobials. Additionally, the FDA has initiated consultations with stakeholders to refine its proposed framework for assessing the human health impact of antimicrobials that may be used in foodproducing animals.

Research. Research will be provided to the community with new information and technologies, including genetic blueprints for various microbes, to identify targets for desperately needed new diagnostics, treatments, and vaccines that could assist in preventing the emergence and spread of resistant pathogens. The NIH plans to develop clinical studies to test new antimicrobials and novel approaches to treating and preventing infections caused by resistant pathogens. The NIH continues to encourage and facilitate new rapid diagnostic methods and will pursue their development and evaluate their ultimate impact in the context of antimicrobial resistance.

Product development. To identify and publicize priority health needs for new products that prevent resistance or treat resistant infections, HHS plans to create an Interagency Antimicrobial Product Development Working Group. Once formed, this group also will consult with stakeholders and economic consultants to identify incentives that encourage this kind of product development.

FROM: Centers for Disease Control and Prevention. A Public Health Action Plan to Combat Antimicrobial Resistance. CDC's antimicrobial resistance web site, http://www.cdc.gov/drugresistance.

\section{VRE Colonization in Liver and Kidney Transplant Recipients}

At Mayo Medical Center (Rochester, MN), surveillance rectal (and other-site) cultures have been collected routinely from liver transplant recipients as part of a selective bowel decontamination program. Beginning in 1995, vancomycin-resistant Enterococcus (VRE) colonization and infection were identified in Mayo Clinic liver and kidney transplant patients through our surveillance cultures. Patel and colleagues conducted a study to describe the natural history of VRE colonization in this patient population. Fifty-two patients with VRE colonization (predominantly with a single $\operatorname{van} \mathrm{B}$ clone) were identified from September 1995 through December 1997. Five hundred ninety cultures were reviewed for this study (mean, 11.3 cultures/patient). The median time from initial VRE colonization to the last surveillance culture obtained was 306 (range, 1-1,393) days.

VRE infection was documented in 6 patients (11.3\%). Eighteen patients (35\%) met the criteria for clearance of VRE colonization, defined as VRE-negative rectal-culture results on at least three consecutive occasions greater than 1 week apart. However, VRE was detected on sub- sequent surveillance cultures from 2 of these patients (11\% relapse rate). Of the remaining 34 patients, 16 remained colonized with VRE, and 18 did not meet the definition for clearance of VRE colonization because of incomplete follow-up.

This study documents that VRE colonization usually persists for months to years in liver and kidney transplant patients.

FROM: Patel R, Allen SL, Manahan JM, Wright AJ, Krom RA, Wiesner RH, et al. Natural history of vancomycin-resistant enterococcal colonization in liver and kidney transplant recipients. Liver Transpl 2001;7:27-31.

\section{VRE Among Chronic Hemodialysis Patients}

D'Agata and coinvestigators from Vanderbilt University School of Medicine, Nashville, Tennessee, conducted a study to determine the prevalence and rate of acquisition of vancomycin-resistant enterococci (VRE) among patients undergoing chronic (ie, long-term) hemodialysis who were admitted to a tertiary-care center. Serial rectal cultures for VRE were performed at hospital admission and every 5 days until hospital discharge. A total of $7(6 \%)$ of the 119 patients were colonized with VRE at admission. Six (19\%) of the 32 patients who remained in the hospital $\geqslant 4$ days acquired VRE.

A nonambulatory status was significantly associated with colonization at admission (odds ratio, 9.7; 95\% confidence interval $\left.\left[\mathrm{CI}_{95}\right], 1.8-53 ; P=.01\right)$, and vancomycin exposure was significantly associated with VRE acquisition (relative risk, $1.8 ; \mathrm{CI}_{05}, 1.1-2.9 ; P=.02$ ). All patients acquired VRE from epidemiologically linked dialysis patients colonized with similar VRE genotypes. Hospital acquisition of VRE contributes substantially to the increasing prevalence of VRE in the chronic hemodialysis patient population.

FROM: D'Agata EM, Green WK, Schulman G, Li H, Tang YW, Schaffner W. Vancomycin-resistant enterococci among chronic hemodialysis patients: a prospective study of acquisition. Clin Infect Dis 2001;32:23-29.

\section{Typing of Coagulase-Negative Staphylococci From Blood Cultures}

Seo and coinvestigators from Beth Israel Deaconess Medical Center and Harvard Medical School, Boston, conducted a study to determine whether a blood culture that contains coagulase-negative staphylococci represents bacteremia or contamination. They compared moleculartyping results of coagulase-negative staphylococcal blood culture isolates with clinical criteria for true bacteremia. Pulsed-field gel electrophoresis and arbitrarily primed (AP) polymerase chain reaction (PCR) were used to determine whether patients with two or more blood cultures with coagulase-negative staphylococcal isolates had the same strain of organism in each culture (same strain bacteremia). They evaluated three different clinical criteria for bacteremia: whether the patient received more than 4 days 